\title{
Percepción de Autoeficacia en la Solución de Problemas y Comunicación Científica en Universitarios de Ingeniería y Ciencias Sociales
}

\author{
Martha Ornelas $^{(1)}$, Humberto Blanco(1)*, Jesús Viciana ${ }^{(2)}$, Judith M. Rodríguez ${ }^{(1)}$ \\ (1)Universidad Autónoma de Chihuahua. Facultad de Ciencias de la Cultura Física. DES Salud CAs 101 y \\ 121. Calle Escorza 900, CP 31000 Chihuahua, Chih.- México. (e-mail: mornelas@uach.mx, \\ hblanco@uach.mx, jurodrig@uach.mx) \\ (2)Universidad Granada. Facultad de Ciencias de la Actividad Física y del Deporte. Carretera de Alfacar, s/n \\ CP: 18071 Granada, España. (e-mail: jviciana@ugr.es) \\ * Autor a quien debe ser dirigida la correspondencia
}

Recibido Dic. 29, 2014; Aceptado Ene. 29, 2015; Versión final recibida Feb. 8, 2015

\begin{abstract}
Resumen
El objetivo de esta investigación consistió en comparar los perfiles de autoeficacia académica de universitarios de ingeniería y ciencias sociales. La muestra fue de 1181 participantes; 592 de Ingeniería y 589 de Ciencias Sociales, con edad media de aproximadamente 18 años. El abordaje adoptado se enmarcó dentro de un enfoque cuantitativo con un diseño descriptivo tipo encuesta. Todos los participantes contestaron la escala autoeficacia en la solución de problemas y comunicación científica. Los resultados de los análisis de varianza mostraron que los perfiles de autoeficacia percibida en la solución de problemas y en comunicación científica son muy similares entre los alumnos de ciencias sociales y de ingeniería en los cuatro índices estudiados. Por ello, la idea de que a las licenciaturas de ciencias sociales llegan alumnos de "menor calidad" que a las ingenierías, es un prejuicio; al menos a lo que autoeficacia percibida se refiere.
\end{abstract}

Palabras clave: creencias del estudiante, educación superior, habilidad percibida, características del estudiante.

\section{Perceived self-efficacy in problem solving and communication in university students of engineering and social sciences}

\begin{abstract}
The purpose of the present study was to compare the profiles of perceived self-efficacy in problem solving and communication between engineering and social sciences university students. A sample of 1181 participants was considered; 592 from engineering and 589 from social sciences, with average age of 18 years. A quantitative approach with a descriptive and transversal survey design was used. All the participants completed the Self-efficacy in problem solving and scientific communication Scale. The results of the analysis of variance showed that perceived self-efficacy profiles in problem solving and in scientific communication are very similar among students of social sciences and engineering in the four indices studied. Therefore the idea that to university careers in social sciences arrive students of "lower quality" that the engineering's is a prejudice, at least according to the perceived self-efficacy.
\end{abstract}

Keywords: student's beliefs, higher education, perceived ability, student characteristics 


\section{INTRODUCCIÓN}

El término autoeficacia se puede definir como los juicios que cada individuo hace sobre sus capacidades, en base a los cuales organizará y ejecutará sus actos de modo que le permitan alcanzar el rendimiento deseado (Bandura, 1997); o como plantea Pajares (2006), las creencias que tiene la persona sobre sus capacidades para organizar y ejecutar caminos para la acción requeridos en situaciones esperadas o basadas en niveles de rendimiento. De acuerdo con Pajares (2006), las creencias que los jóvenes tienen sobre su capacidad para tener éxito en lo que emprenden representan fuerzas vitales en sus subsecuentes éxitos o fracasos; por lo que seguramente van a seleccionar las tareas y actividades que creen están dentro de su capacidades y evitar aquellos que creen que están más allá de su percepción de competencia.

La teoría social-cognitiva propuesta por Bandura (1986) sobre el funcionamiento humano que hace hincapié en el papel de las creencias personales. En esta perspectiva social cognitiva, los individuos son vistos como proactivos, autorganizados, autorreflexivos y autorregulados, en lugar de ser organismos conducidos por instintos internos escondidos o modulados por fuerzas ambientales. El pensamiento y la acción humanos son vistos como el producto de una interacción dinámica de las influencias personales, conductuales y ambientales; como las personas interpretan los resultados de sus propias acciones informa y altera sus entornos y los factores personales que poseen, los cuales, a su vez, informan y modifican las acciones futuras. Bandura establece, en su determinismo recíproco, una interacción dinámica entre factores. Es así que a) factores personales en la forma de afectos, cogniciones y eventos biológicos, b) el comportamiento y c) fuerzas ambientales crean interacciones que producen una reciprocidad tríadica.

De esta manera La teoría social-cognitiva enfatiza el papel de los fenómenos autorrefenciales como el medio por el cual el ser humano es capaz de actuar en su ambiente y por consecuencia transformarlo, las personas crean y desarrollan autopercepciones acerca de su capacidad, mismas que se convierten en los medios por los cuales siguen sus metas y toman sus decisiones (Gutiérrez et al., 2011). Esto es, la forma en que la gente actúa es en parte producto de la intervención de sus creencias acerca de lo que es capaz de realizar.

La investigación empírica ha demostrado, de manera amplia, que la autoeficacia resulta ser más predictiva del rendimiento académico que otras variables cognitivas (Bandura, 1982), también que logra predecir el éxito posterior (Bandura, 1997; Ornelas et al., 2012) y que es un importante mediador cognitivo de competencia y rendimiento (Vera et al., 2011) en cuanto favorece los procesos cognoscitivos (Carbonero y Merino, 2008; Ornelas et al., 2011). El comportamiento de las personas, según Bandura (1997), puede ser mejor predicho por las creencias que los individuos tienen acerca de sus propias capacidades que por lo que en verdad pueden hacer, puesto que estas percepciones contribuyen a delinear qué es lo que las personas hacen con las habilidades y el conocimiento que poseen (Adeyemo, 2007; Blanco et al., 2011). Dicha autopercepción, denominada autoeficacia, ejerce una profunda influencia en la elección de tareas y actividades, en el esfuerzo y perseverancia de las personas cuando se enfrentan a determinados retos e incluso en las reacciones emocionales que experimentan ante situaciones difíciles (Vera et al., 2011). En definitiva, las creencias de autoeficacia representan un mecanismo cognitivo que media entre el conocimiento y la acción y que determina, junto con otras variables, el éxito de las propias acciones (Carbonero y Merino, 2008; Ornelas et al., 2011).

Como una muestra de la importancia de la autoeficacia en el ámbito académico, podemos decir que esta revela por qué las personas con el mismo nivel de habilidad y conocimiento presentan conductas y/o resultados diferentes, o por qué las personas actúan en disonancia con sus habilidades (Bandura, 1982; Pérez et al., 2011). Lo anterior se explica porque el rendimiento académico adecuado también depende de la eficacia percibida para manejar demandas académicas exitosamente. Por ello, las creencias de autoeficacia en la propia capacidad son imprescindibles para dominar las actividades académicas; ya que los estudiantes que confían en sus capacidades se sienten más motivados para alcanzar sus metas (Á. Blanco, 2010). Así mismo, las personas que dudan de sus capacidades pueden creer que las cosas son más difíciles de lo que realmente son, creencia que genera tensión, depresión y una visión estrecha para resolver problemas (Vera et al., 2011). Se ha evidenciado que un bajo nivel de autoeficacia puede ser responsable no sólo de disminución del rendimiento escolar e interés hacia el estudio, sino también de comportamientos inadaptados en jóvenes (Zimmerman y Kitsantas, 2005), de ahí la importancia de que la educación fortalezca el desarrollo de la competencia académica en el estudiante y fomente habilidades que le permitan creer en sus propias capacidades (Carbonero y Merino, 2008; Ornelas et al., 2011).

Este trabajo es fundamentalmente un estudio de tipo descriptivo que intenta comparar los perfiles de autoeficacia académica percibida, adquiridos en el nivel educativo previo, de alumnos universitarios de ingeniería y ciencias sociales. El estudio pretende, como investigación aplicada, aportar información que se traduzca en una práctica educativa de mayor calidad en el contexto de atención a la diversidad; 
contribuyendo al saber pedagógico en el esclarecimiento de los factores que conforman un modelo rendimiento escolar y desarrollo humano integral; bajo la premisa de que, darse a la tarea de mejorar la percepción de ser capaz en quien aprende es un objetivo educacional valioso, bajo el supuesto implícito de que su potenciación servirá como vehículo para la mejora de otros resultados tales como el logro académico y la autoestima. Tomando en cuenta que la continua conciencia de fracaso reduce las expectativas de éxito y no favorece en ningún modo ni el aprendizaje ni el desarrollo personal. El presente estudio contribuye a aportar evidencias y datos que propician la intervención educativa dentro de una perspectiva educativa de atención a la diversidad en el aula.

\section{METODOLOGÍA}

\section{Participantes}

La muestra correspondió a 1181 participantes (aproximadamente el $50 \%$ de los inscritos en primer semestre), $592(50,1 \%)$ estudiantes de ingeniería y 589 (49,9\%) estudiantes de ciencias sociales. Se utilizó un muestreo por conveniencia, con el fin de abarcar la representatividad de las diferentes licenciaturas de ambas áreas (ingeniería y ciencias sociales) que se ofrecen en la Universidad Autónoma de Chihuahua: (1) Muestra alumnos de ingeniería constituida por 592 participantes; $143(24.2 \%)$ mujeres y 449 (75.8\%) hombres. Con un rango de edad entre los 17 y 20 años, una media de 18.16 y una desviación estándar de 0.72 años; y (2) Muestra alumnos de ciencias sociales compuesta por 589 participantes; 376 (63.8\%) mujeres y $213(36.2 \%)$ hombres. Con un rango de edad entre los 17 y 20 años, una media de 18.24 y una desviación estándar de 0.73 años.

\section{Instrumento}

La Escala Autoeficacia en la Solución de Problemas y Comunicación Científica es una encuesta tipo Likert, asistida por computadora de 11 ítems (Aguirre et al., 2012) que corresponde a cada uno de los principales dominios incluidos en las competencias básicas sobre solución de problemas y comunicación de los currículos de todas las licenciaturas que se imparten en la Universidad Autónoma de Chihuahua. Donde el encuestado responde, en una escala de 0 a 10, que tan capaz se siente, que tanto interés tiene y si se esfuerza en cambiar que tan capaz sería en cada uno de los dominios (ítems) de las competencias Solución de Problemas y Comunicación Científica (factores de la escala). Cada sujeto respondió a cada uno de los ítems del instrumento en tres escenarios:

i) Escenario de capacidad percibida, respondiendo en el contexto: que tan capaz me siento para... desempeñarme en cada uno de los dominios de las competencias antes mencionadas.

ii) Escenario de interés en ser capaz, respondiendo en el contexto: que tanto interés tengo en ser capaz de... desempeñarme en cada uno de los dominios de las competencias antes mencionadas.

iii) Escenario de cambio en ser capaz de, respondiendo en el contexto: si me esfuerzo en cambiar, que tan capaz sería para... desempeñarme en cada uno de los dominios de las competencias antes mencionadas.

Para luego a partir de sus respuestas obtener 4 índices: 1.- Autoeficacia percibida, obtenida a partir de las respuestas al escenario de capacidad percibida; 2.- Autoeficacia deseada, obtenida a partir de las respuestas al escenario interés en ser capaz; 3.- Autoeficacia alcanzable, obtenida a partir de las respuestas al escenario de cambio en ser capaz de; y 4.- Posibilidad de mejoría en la autoeficacia percibida, obtenida a través de la diferencia entre el índice 3 y 1 (Autoeficacia alcanzable menos Autoeficacia percibida).

La estructura de dos factores (Tabla 1): Solución de Problemas y Comunicación Científica, para esta escala, atendiendo a criterios estadísticos y sustantivos, ha mostrado adecuados indicadores de ajuste, de fiabilidad y de validez (Aguirre et al., 2012; Elosua y Zumbo, 2008; Thompson, 2004).

\section{Diseño}

Se utilizó un enfoque cuantitativo con un diseño descriptivo y transversal tipo encuesta (Hernández et al., 2010). La variable independiente fue Tipo de Licenciatura (estudiantes de ingeniería y ciencias sociales) y las variables dependientes el promedio de las puntuaciones obtenidas en los cuatro índices para los factores Solución de Problemas y Comunicación Científica.

\section{Procedimiento}

Se invitó a participar en el estudio a los alumnos de primer semestre de las licenciaturas de ciencias de la salud y ciencias sociales que se ofrecen en la Universidad Autónoma de Chihuahua; los que aceptaron 
participar firmaron la carta de aceptación correspondiente. Luego se aplicó el instrumento antes descrito por medio de una computadora personal utilizando el módulo administrador del instrumento del editor de escalas versión 2.0 (Blanco et al., 2013), en una sesión de aproximadamente 30 minutos; en los centros de cómputo de las unidades académicas participantes. Al inicio de cada sesión se hizo una pequeña introducción sobre la importancia de la investigación y de cómo acceder al instrumento; las instrucciones de como responder se encontraban en las primeras pantallas; antes del primer reactivo del instrumento. Al término de la sesión se les agradeció su participación. Una vez aplicado el instrumento se procedió a recopilar los resultados por medio del módulo generador de resultados del editor de escalas versión 2.0 (Blanco et al., 2013).

Tabla 1: Ítems de escala autoeficacia en la solución de problemas y comunicación científica, agrupados por factor

\begin{tabular}{|c|c|}
\hline Factor & Ítem \\
\hline $\begin{array}{l}\text { Solución de } \\
\text { Problemas }\end{array}$ & $\begin{array}{l}1 \text { Aplicar las diferentes técnicas de observación para la solución de problemas } \\
3 \text { Distinguir los diferentes tipos de sistemas } \\
5 \text { Emplear diferentes métodos para establecer alternativas de solución de problemas } \\
6 \text { Aplicar el enfoque sistémico en diversos contextos } \\
9 \text { Emplear la estadística en la interpretación de resultados y construcción de conocimiento } \\
11 \text { Analizar los diferentes componentes de un problema y sus interrelaciones }\end{array}$ \\
\hline $\begin{array}{l}\text { Comunicación } \\
\text { Científica }\end{array}$ & $\begin{array}{l}2 \text { Recopilar analizar y aplicar información de diferentes fuentes } \\
4 \text { Manejar recursos documentales y electrónicos que apoyan a la comunicación y búsqueda de } \\
\text { información } \\
7 \text { Desarrollar escritos a partir de procesos de investigación } \\
8 \text { Manejar y aplicar paquetes computacionales para desarrollar documentos, presentaciones y } \\
\text { bases de información } \\
10 \text { Leer e interpretar textos }\end{array}$ \\
\hline
\end{tabular}

\section{Análisis de datos}

Se realizaron análisis estadísticos descriptivos (medias y desviaciones estándar) para todas las variables. Posteriormente, después de verificar que los datos cumplían con los supuestos de los análisis estadísticos paramétricos, se llevó a cabo un análisis multivariante de varianza (MANOVA), seguido por los análisis de varianza univariados (ANOVAs), para examinar las diferencias entre los alumnos de ciencias sociales y de ingeniería en cuanto a su autoeficacia percibida en los factores Solución de Problemas y Comunicación Científica. Por otra parte, el tamaño del efecto se estimó mediante el eta cuadrado $\left(\eta^{2}\right)$. La fiabilidad o consistencia interna de la cada variable se estimó utilizando el coeficiente alfa de Cronbach con un intervalo de confianza del 95\%. Todos los análisis estadísticos se realizaron con el programa SPSS versión 20.0 para Windows. El nivel de significación estadística se estableció en p=0.05.

\section{RESULTADOS Y DISCUSIÓN}

\section{Solución de Problemas}

La Tabla 2 muestra los valores de las medias y las desviaciones estándar de la autoeficacia en el factor solución de problemas, así como los resultados del MANOVA y los subsiguientes ANOVAs. Los resultados del MANOVA indicaron diferencias significativas globales de acuerdo a la variable tipo de licenciatura en las puntuaciones de autoeficacia del factor solución de problemas (Wilks' $\lambda=.992 ; p<.05 ; \eta^{2}=.008$ ). En la Tabla 2, los valores descriptivos se presentan como media (desviación estándar). Posteriormente, los resultados de los ANOVAs mostraron que los estudiantes de ciencias sociales reportaron, en este factor, mayor posibilidad de mejoría en su percepción de autoeficacia $\left(F_{1}=4.894, p<.05\right)$ que los estudiantes de ingeniería y sin diferencias significativas en autoeficacia percibida, autoeficacia deseada y alcanzable. Finalmente, la consistencia interna (confiabilidad) del factor solución de problemas fue muy alta: autoeficacia percibida .894 (.884-.903), autoeficacia deseada .903 (.894-.911) y autoeficacia alcanzable .905 (.896-.913). 
Tabla 2: Resultados MANOVA y ANOVAs para las diferencias entre alumnos de ingeniería y ciencias sociales en los cuatro índices de autoeficacia para el factor solución de problemas.

\begin{tabular}{|l|c|c|c|c|c|}
\hline & $\begin{array}{c}\text { Ciencias Sociales } \\
(\mathrm{n}=589)\end{array}$ & $\begin{array}{c}\text { Ingeniería } \\
(\mathrm{n}=592)\end{array}$ & $F$ & $p$ & $\eta^{2}$ \\
\hline Autoeficacia percibida & & & 3.316 & $<.05$ & .008 \\
\hline Autoeficacia deseada & $7.46(1.35)$ & $7.58(1.26)$ & 2.661 & $>.05$ & .000 \\
\hline Autoeficacia alcanzable & $8.64(1.12)$ & $8.76(1.16)$ & 3.153 & $>.05$ & .000 \\
\hline $\begin{array}{l}\text { Posibilidad de mejoría en la } \\
\text { autoeficacia percibida }\end{array}$ & $8.97(0.93)$ & $8.98(0.98)$ & 0.005 & $>.05$ & .000 \\
\hline
\end{tabular}

\section{Comunicación Científica}

La Tabla 3 muestra los valores de las medias y las desviaciones estándar de la autoeficacia en el factor comunicación, así como los resultados del MANOVA y los subsiguientes ANOVAs. En la Tabla los valores descriptivos se presentan como media (desviación estándar). Los resultados del MANOVA no indicaron diferencias significativas globales de acuerdo a la variable tipo de licenciatura en las puntuaciones de autoeficacia del factor comunicación científica (Wilks' $\lambda=.996 ; p>.05 ; \eta^{2}=.004$ ). Posteriormente, los resultados de los ANOVAs no mostraron ninguna diferencia significativa entre los estudiantes de ciencias sociales y de ingeniería en los cuatro índices de autoeficacia del factor comunicación científica estudiados. Finalmente, la consistencia interna (confiabilidad) del factor comunicación científica fue muy alta: autoeficacia percibida $.832(.816-.847)$, autoeficacia deseada $.857(.844-.870)$ y autoeficacia alcanzable .853 (.839-.866).

Tabla 3: Resultados MANOVA y ANOVAs para las diferencias entre alumnos de ingeniería y ciencias sociales en los cuatro índices de autoeficacia para el factor comunicación científica.

\begin{tabular}{|l|c|c|c|c|c|}
\hline & $\begin{array}{c}\text { Ciencias Sociales } \\
(\mathrm{n}=589)\end{array}$ & $\begin{array}{c}\text { Ingeniería } \\
(\mathrm{n}=592)\end{array}$ & $F$ & $p$ & $\eta^{2}$ \\
\hline Autoeficacia percibida & $7.80(1.31)$ & $7.79(1.30)$ & 0.002 & $>.05$ & .000 \\
\hline Autoeficacia deseada & $8.94(1.06)$ & $8.90(1.15)$ & 0.289 & $>.05$ & .000 \\
\hline Autoeficacia alcanzable & $9.20(0.84)$ & $9.11(0.95)$ & 2.475 & $>.05$ & .002 \\
\hline $\begin{array}{l}\text { Posibilidad de mejoría en la } \\
\text { autoeficacia percibida }\end{array}$ & $1.40(0.96)$ & $1.32(0.94)$ & 2.057 & $>.05$ & .002 \\
\hline
\end{tabular}

En relación al factor Solución de Problemas el cual implica ser capaz de aplicar las diferentes técnicas de observación para la solución de problemas; distinguir los diferentes tipos de sistemas; emplear diferentes métodos para establecer alternativas de solución de problemas; aplicar el enfoque sistémico en diversos contextos; emplear la estadística en la interpretación de resultados y construcción de conocimiento; así como, analizar los diferentes componentes de un problema y sus interrelaciones; aunque los alumnos de ciencias sociales se perciben con mayor posibilidad de mejoría en su autoeficacia que los alumnos de ingeniería no existen diferencias en su autoeficacia percibida, deseada y alcanzable. En cuanto al factor Comunicación Científica, es decir, recopilar analizar y aplicar información de diferentes fuentes; manejar recursos documentales y electrónicos que apoyan a la comunicación y búsqueda de información; desarrollar escritos a partir de procesos de investigación; manejar y aplicar paquetes computacionales para desarrollar documentos, presentaciones y bases de información; además de leer e interpretar textos; los resultados obtenidos muestran que los perfiles de su autoeficacia percibida, deseada y alcanzable, así como la posibilidad de mejoría en su autoeficacia son equivalentes. Resultados que en general concuerda con los obtenidos por Peinado (2011) en un estudio similar.

Por último, tomando en cuenta que la investigación empírica ha demostrado, de manera amplia, que la autoeficacia resulta ser más predictiva del rendimiento académico que otras variables cognitivas (Bandura, 1982), que logra predecir el éxito posterior (Bandura, 1997) y que es un importante mediador cognitivo de competencia y rendimiento (Vera et al., 2011) en cuanto favorece los procesos cognoscitivos (Carbonero y Merino, 2008; Ornelas et al., 2011) concluimos que darse a la tarea de mejorar la percepción de ser capaz en quien aprende es un objetivo educacional valioso, bajo el supuesto implícito de que su potenciación 
servirá como vehículo para la mejora de otros resultados tales como el logro académico y la autoestima. Tomando en cuenta que la continua conciencia de fracaso reduce las expectativas de éxito y no favorece en ningún modo ni el aprendizaje ni el desarrollo personal.

\section{CONCLUSIONES}

De los resultados mostrados, de su análisis y de su discusión, se pueden obtener las siguientes conclusiones:

1) Los alumnos de ciencias sociales y de ingeniería muestran perfiles de autoeficacia muy similares en los factores de solución de problemas y comunicación científica; por lo que la idea de que a las licenciaturas de ciencias sociales llegan alumnos de "menor calidad" que a las de las ingenierías, es solo un prejuicio; al menos a lo que autoeficacia percibida se refiere (H. Blanco, 2010; Ornelas et al., 2012).

2) En las escalas de autoeficacia habitualmente se les pregunta a las personas acerca de sus capacidades operativas en el presente, no acerca de sus capacidades potenciales o sobre sus capacidades futuras esperadas (Bandura, 2006). Mientras que en la Escala Autoeficacia en la Solución de Problemas y Comunicación Científica utilizada en la presente investigación, además de que el estudiante responde que tan capaz se siente actualmente, manifiesta también que tanto interés tiene y si se esfuerza en cambiar que tan capaz sería en cada uno de los ítems. Lo que permite, entre otras cosas, generar disonancia cognoscitiva en el estudiante en relación a su autoeficacia percibida en el presente, el nivel de autoeficacia deseado y la posibilidad de mejoría en la autoeficacia percibida. La disonancia así generada, incrementa la motivación por realizar actividades dirigidas a la reducción de la misma; lo cual puede ser aprovechado por el docente o tutor académico del estudiante para que esté establezca metas de logro para aumentar su eficacia, aproximándola a la deseada o alcanzable y de esa manera potenciar su aprendizaje.

\section{REFERENCIAS}

Adeyemo D. A., Moderating Influence of Emotional Intelligence on the Link Between Academic Self-efficacy and Achievement of University Students, Psychology Developing Societies, 19(2), 199-213, (2007).

Aguirre J. F., Muñoz F., De Rueda B., Blanco J. R., Composición Factorial de una Escala de Autoeficacia en la Solución de Problemas y Comunicación en Universitarios de Ciencias Sociales, doi: 10.4067/S071850062012000500004, Formación Universitaria, 5(5), 27-38, (2012).

Bandura A., Self-efficacy mechanism inhuman agency, American Psychologist, 37(2), 122-147, (1982).

Bandura A., Self-efficacy: The exercise of Control, Freeman, New York, (1997).

Bandura A., Social foundations of thought and action: A social cognitive theory, Prentice Hall, Englewood Cliffs, NJ, (1986).

Bandura A., Guide for constructing self-efficacy scales, En Self-efficacy beliefs of adolescents, por F. Pajares, Urdan T. C., pp 307-337 Age Publishing, Inc., Greenwich, (2006).

Blanco Á., Creencias de autoeficacia de estudiantes universitarios: un estudio empírico sobre la especificidad del constructo, RELIEVE, 16(1), 1-28, (2010).

Blanco H., Autoeficacia percibida en conductas académicas y cuidado de la salud en alumnos de primer ingreso a la universidad autónoma de chihuahua. Un estudio comparado respecto a los alumnos universitarios de educación física, Universidad de Granada, España, (2010).

Blanco H., Martínez M., Zueck M. d. C., Gastélum G., Análisis psicométrico de la escala autoeficacia en conductas académicas en universitarios de primer ingreso, Actualidades Investigativas en Educación, 11(3), 1-27, (2011).

Blanco H., Ornelas M., Tristán J. L., Cocca A., Mayorga-Vega D., López-Walle J., et al., Editor for creating and applying computerise surveys, doi: http://dx.doi.org/10.1016/j.sbspro.2013.12.105, Procedia Social and Behavioral Sciences, 106, 935-940, (2013).

Carbonero M. Á., Merino E., Autoeficacia y madurez vocacional, Psicothema, 16(2), 229-234, (2008). 
Elosua P., Zumbo B. D., Coeficientes de fiabilidad para escalas de respuesta categórica ordenadas, Psicothema, 20(4), 896-901, (2008).

Gutiérrez M., Ampara E., Carminal P., Relaciones entre empatía, conducta prosocial, agresividad, autoeficacia y responsabilidad personal y social de los escolares, Psicothema, 23(1), 13-19, (2011).

Hernández R., Fernández C., Baptista P., Metodología de la investigación, McGraw- Hill, México, (2010).

Ornelas M., Blanco H., Gastélum G., Chávez A., Autoeficacia Percibida en la conducta Académica de Estudiantes Universitarias, doi: 10.4067/S0718-50062012000200003, Formación Universitaria, 5(2), 17-26, (2012).

Ornelas M., Blanco H., Rodríguez J. M., Flores F. J., Análisis psicométrico de la escala autoeficacia en conductas de cuidado de la salud física en universitarios de primer ingreso, doi: 10.4067/S071850062011000600004, Formación Universitaria, 4(6), 21-34, (2011).

Pajares F., Self-Efficacy During Childhood and Adolescence: Implications for Teachers and Parents, En Selfefficacy Beliefs of Adolescents, por F. Pajares, Urdan T. C., pp 339-367 Information Age Publishing, Greenwich, CT, (2006).

Peinado J. E., Efecto de la disciplina académica sobre la percepción de las competencias básicas en universitarios chihuahuenses, Universidad de Granada, España, (2011).

Pérez E., Lescano C., Heredia D., Zalazar P., Furlám L., Martínez M., Desarrollo y análisis psicométricos de un inventario de autoeficacia para inteligencias múltiples en niños argentinos Psicoperspectivas, 10(1), 169 189, (2011).

Thompson B., Exploratory and Confirmatory Factor Analysis. Understanding concepts and applications. , American Psychological Association, Washington, D C, (2004).

Vera M., Salanova M., Martín-del-Río B., Self-efficacy among university faculty: how to develop an adjusted scale, Anales de Psicología, 27(3), 800-807, (2011).

Zimmerman B., Kitsantas A., Homework practice and academic achievement. The mediating role of selfefficacy and perceived responsibility beliefs, Contemporary Educational Psychology, 30(4), 397-417, (2005). 
